\title{
Computerized Training for Neuroplasticity and Cognitive Improvement
}

\author{
https://doi.org/10.3991/ijep.v9i4.10285 \\ Marios A. Pappas ${ }^{(凶)}$, Athanasios S. Drigas \\ National Center for Scientific Research "Demokritos", Attica, Greece \\ mpapdiit.demokritos.gr
}

\begin{abstract}
The research area of brain plasticity studies indicates that individuals can train and improve their cognitive abilities throughout life. In addition, more and more computerized training tools are presented in recent studies. The purpose of this study is to review studies of the last decade in the field of cognitive training using Information and Communication Technologies, to record the cognitive improvement techniques used, as well as to evaluate the effectiveness of these intervention programs. As indicated by the literature review, computer-based tools, mobile training apps and video games could be used in intervention studies for cognitive improvement. In addition, cognitive training techniques seem to be significantly effective for the cognitive improvement of healthy or cognitive impaired individuals.
\end{abstract}

Keywords-Cognitive improvement, neuroplasticity, neurodevelopment, cognitive training

\section{$1 \quad$ Introduction}

Neuroimaging studies have shown that the human brain develops throughout life, especially from birth through adulthood [1]. High plasticity of the human brain indicates that perceptual-cognitive abilities are trainable [2]. Brain plasticity could be considered a memory function that affects the capacity of information processing and the ability to acquire skills $[3,4]$.

Brain plasticity is the basis for the functional recovery on individuals after brain damage, as well as for the reduction of the effects caused by Parkinson's disease, aphasia, Alzheimer's disease, etc. $[5,6,7,8]$. There is evidence that music making, as well as learning how to play a musical instrument could influence brain plasticity across the life span $[9,10]$. In addition, recent studies show that physical activity and especially aerobic training, as well as resistance training, could promote cognitive brain plasticity in seniors $[11,12]$. Pareja-Galeano et al (2013) emphasized the significant effect of exercise training on the increase of neuroplasticity-related proteins on healthy adolescents [13]. Furthermore, meditation-based training displays neuroplasticity in the attentional networks and is also considered an effective technique for the improvement of cognitive skills [14]. 
Most parents believe that television viewing could enhance cognitive development of their children. Studies show that this is not true for very young children. The study of Zimmerman \& Christakis (2005) showed that television viewing before age 3 years could act negatively to the cognitive outcomes at the age of 6 and 7 years [15]. On the other hand, television viewing from 3 to 5 years old children, could enhance their reading recognition and short-term memory. In addition, a study with 1,008 parents of children under the age of 2 years in Washington and Minnesota, revealed that there is strong negative correlation between viewing baby videos and vocabulary acquisition [16]. According to Mar, Tackett \& Moore (2010) exposure to children's movies is a reliable predictor of children's theory of mind (i.e. the ability of understanding mental stages of others). However, based on their study, the same could not be said for children's television shows [17]. Analyzing data from 678 New York families, Johnson, Cohen, Kasen \& Brook (2007) showed that extensive television viewing (more than 3 hours per day) during adolescence, could increase the risk for attention and learning difficulties [18].

During the last decade, various digital cognitive training tools have been developed, incorporating traditional training techniques. The purpose of this study is to evaluate the effectiveness of the use of Information and Communication Technologies, such as computer-based intervention tools, mobile apps and video games, on intervention programs for cognitive improvement. In addition, this study aims to analyze the cognitive training techniques which are considered, based on the literature, as the most effective for cognitive improvement. For this reason, we analyze the most representative studies on this topic, published during the last decade.

\section{State-of-the-Art}

\subsection{Computerized cognitive training}

CALM Tools for Living is a set of web-based innovative tools, which use cognitive behavioral therapy (CBT) principles for anxiety disorders. Its eight modules are designed to enhance self-monitoring, fear hierarchy, breathing, cognitive restructuring, exposure to external cues, exposure to stimuli and relapse prevention. This computer assisted program was positively rated by clinicians and patients, as intervention resulted significant reduction in anxiety symptoms [19].

Mishra, Sagar, Joseph, Gazzaley \& Merzenich (2016) applied online neuroplasticity-based cognitive training on children with Attention Deficit Hyperactivity Disorder (ADHD). Intervention, which was based on 25 online cognitive exercise modules, led to reduction of distractor-based errors for the participants, as well as to improvement of their scores on response inhibition and Stroop interference tests [20].

A study with thirty older adults revealed that non-action video games could enhance cognitive their skills. Specifically, results of the study showed that training for 20 one-hour sessions with ten cognitive-based video games from the online platform Lumosity, improved participants processing speed, sustained attention, immediate and 
delayed visual memory and wellbeing dimensions. However, the training did not improve spatial working memory and executive control [21].

In a study with 42 older adults (mean age $=67.38$ years), members of the experimental group participated in a digital inclusion workshop, containing training on the basics of computing and Internet browsing. Training on computers and Internet searching resulted significant improvement on participants' executive functions, as well as on their language and memory skills [22].

Ball, Edwards \& Ross (2007) examined the impact of speed of processing training, which involves computer-based nonverbal exercises, on cognitive and everyday abilities of seniors [23]. Exercises that are included in the speed of processing training are based on target detection, identification, discrimination and localization in limited time. According to the results, this training protocol could improve speed of processing, as well as executive function and memory of adults from 55 to 95 years old.

Smith et al (2009) proposed a computer-based cognitive training program containing auditory information processing exercises for the improvement of untrained measures of memory and attention [24]. Discrimination, recognition, matching and reconstruction of auditory stimuli could lead to plastic changes in brain systems and thus result in improvement of memory and attention, as revealed by delayed recall, digits backwards and letter-number sequencing tasks.

Fisher, Holland, Merzenich \& Vinogradov (2009) used a neuroplasticity-based, computerized auditory training program in order to enhance memory and cognitive skills of people with schizophrenia [25]. Results revealed significant improvement of participants' verbal working memory and global cognition.

Voelbel, Ceceli, Georgieva, Tortarolo \& Lindsey (2014) evaluated the effect of computerized neuroplasticity-based training on adults with traumatic brain injury (TBI). According to the results, there was significant improvement of participants' processing speed and task switching ability [26].

In a study with 31 schizophrenia patients, Subramaniam et al (2012) evaluated the impact of intensive computerized training on patients' neural activity [27]. Patients who trained for 10 weeks with computerized auditory/verbal, visual and social processing exercises, showed significant improvement in reality monitoring, as well as an increase in medial prefrontal cortex (mPFC) activation.

\section{$2.2 \quad$ Video games}

The study of Clemenson and Stark (2015) indicated that playing complex 3D video games like Super Mario 3D World, improves hippocampal-associated memory [28]. On the other hand, training in a 2D game, Angry Birds, did not result in such improvement. Developmental psychologists, during the last years, claim that digital game play contributes to children's and adolescents' cognitive development [29]. A study with 48 participants (mean age 24.1 years) in Berlin, Germany, used Super Mario 64, a 3D platformer game for training, in order to measure plasticity effects on specific brain regions. Results of the study revealed significant increase of gray matter in the right hippocampal formation [30]. In addition, multitasking training with $\mathrm{Neu}$ roRacer, a 3D computer-based video game, had significantly positive effect on sus- 
tained attention and working memory of older adults (mean age $=67.5$ years) [31]. In addition, 54 older adults (mean age $=67.8$ years) were trained with five cognitive online video games for seven weeks. Results showed that seniors playing video games can improve their inhibition and inductive reasoning [32]. Finally, the study of Li, Ngo, Nguyen \& Levi (2011) revealed that playing action video-games, in which players should track fast moving objects, could result visual plasticity in adults with amblyopia, most known as "lazy eye" [33].

\subsection{Mobile training apps}

Mobile apps are considered as effective, low-cost tools for behavioral improvement [34]. Engaging mobile apps (interactive e-books, games, or creating apps) could enhance awareness and goal accomplishment in 4 to 6 years old children [35]. 3125 US citizens (mean age 32.4 years) participated in an online survey regarding their perceptions on brain training mobile apps. Participants claimed that cognitive training apps could improve their thinking, memory, attention, or even their mood [36].

Oiva, an Android mental wellness training app, designed using acceptance and commitment (ACT) principles, seems to improve users' mental wellness, reducing stress levels [37]. Two hundred twenty-seven eighth-grade students in Chile participated in an experimental study. Three mobile serious games were used for students' training: Evolution, a strategy game using 3D graphics and BuinZoo \& Museum, which are trivia games. According to the results, playing mobile serious games benefits problem solving skills and especially plan execution capacities [38]. HealtheBrain is an innovative mobile application, compatible with iPhone and iPad (available on Apple Store), which is based on evidence that physical activity is correlated with cognitive improvement. In particular, HealtheBrain offers square-stepping exercise, a visuospatial working memory task which improves cognitive and executive functioning in older adults [39]. Chittaro \& Sioni (2014) used three versions of a mobile app for breathing training, offering visual and auditory instructions for users, in order to adapt their breathing frequency [40]. During the procedure, researchers collected data about the skin conductance level, the heart rate, the power of frequency band, the respiratory signal to noise ratio, the perceived effectiveness, as well as the relaxation effectiveness. Wave-based visualization provided from the app offered the opportunity for participants to track over time their breathing training and thus enhance their relaxation and reduce their stress levels.

\section{$2.4 \quad$ Neurofeedback training}

Several studies reveal that neurofeedback training (NFT) has positive effect on individuals with behavioral or neurological disorders, such as attention deficit hyperactivity disorder (ADHD), Parkinson's disease, autism, etc. [41, 42, 43, 44]. Electroencephalogram (EEG) alpha activity seems to be highly related to memory performance. In the experiment of Nan et al (2012), consisting 32 participants (mean age $=23.28$ years), neurofeedback training of the upper alpha frequency band, enhanced selfregulation of brain activity, as well as short-term memory performance [45]. In addi- 
tion, the study of Wang and Hsieh (2013) revealed that frontal-midline theta $(\mathrm{fm} \theta)$ training improves attention and working memory performance in the elderly [46]. In addition, the $\operatorname{fm} \theta$ training protocol benefited orienting and executive control of the participants (mean age $=65$ years).

Magnetic resonance imaging (MRI) studies have shown that NFT can lead to changes in white and gray matter [47]. Structural changes in white and gray matter volume, as well as functional reorganization after neurofeedback sessions are indicators of neural plasticity [48]. Furthermore, mental rotation tests have indicated that training with neurofeedback on the upper alpha frequency, could significantly improve cognitive performance [49].

\section{$3 \quad$ Methodology}

The present paper targets to answer the following research questions: -Can ICTbased training be used for cognitive improvement? -Which training techniques can be used for cognitive improvement? -Which technologies can be used for cognitive intervention? This review presents research studies from 2007 to 2018, which contain ICT-based cognitive training for cognitive improvement of the participants. In order to collect published studies from peer-reviewed journals, we searched on the following databases: Academic Search, ERIC, PubMED Central, PsycINFO, CiteSeerX, dblp and Scopus. Search terms used were: neuroplasticity, neuroplasticity training, cognitive improvement, mobile training app, computerized cognitive training, neurofeedback training. The following inclusion criteria were used in order to identify eligible studies:

- Study population consisted of healthy or cognitive impaired individuals

- Any type of computerized cognitive intervention

- Use of subjective outcome measures after the intervention. Furthermore, studies based on the same study population were excluded. After the final selection we extracted data from each study in terms of the purpose, the study population, the intervention design, the cognitive training, as well as the training outcomes.

\section{$4 \quad$ Results}

Figure 1 presents the studies selected for this review. Twenty-two studies were finally included. There were twenty experimental studies and two field studies. Of the selected studies, sixteen studies included a sample of healthy individuals and six studies included a sample of cognitive impaired individuals. The number of healthy participants in the experimental groups varied from 15 [37] to 242 [24], while the number of participants with cognitive impairment varied from 13 [19] to 31 [20]. The mean age of the participants ranged from 5 years [35] to 75.6 years [24]. Nine studies aimed at the improvement of working memory, while four studies aimed at the improvement of participants' attentional skills. In addition, four studies aimed at the reduction of anxiety symptoms, as well as of stress levels, while eight studies aimed 


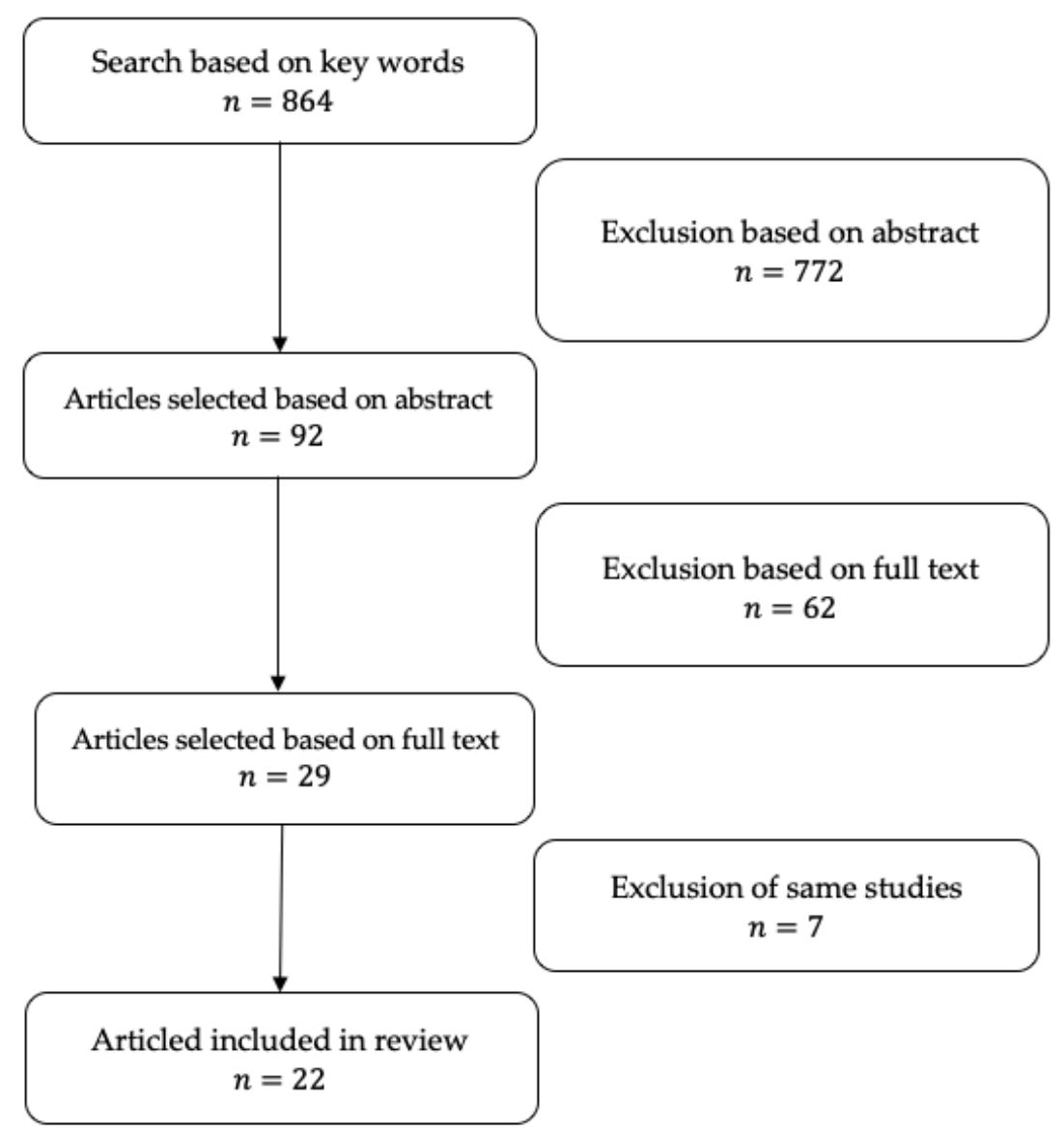

Fig. 1. Selection process for the review.

at the improvement of participants' metacognitive skills such as inhibition, task switching, executive function, inductive reasoning and self-regulation.

Results have shown that the techniques which could enhance cognitive improvement were cognitive behavioral therapy, speed of processing training, auditory training, eye tracking, acceptance and commitment, physical activity, breathing training, visualization and neurofeedback training. Intervention studies for cognitive improvement utilized computerized training tolls, commercial video games, serious games, training mobile apps, as well as neurofeedback equipment. 
Table 1. Study characteristics of selected studies for cognitive improvement.

\begin{tabular}{|c|c|c|c|c|c|}
\hline Study & Purpose & Sample & Intervention & $\begin{array}{c}\text { Cognitive } \\
\text { Training } \\
\text { Protocol } \\
\end{array}$ & Results \\
\hline \begin{tabular}{|l|} 
Ahtinen et al \\
$(2013)$
\end{tabular} & $\begin{array}{l}\text { Effect of mobile } \\
\text { mental wellness } \\
\text { training apps on } \\
\text { stress management }\end{array}$ & $\begin{array}{l}15 \text { working } \\
\text { age partici- } \\
\text { pants }\end{array}$ & $\begin{array}{l}\text { Mental well- } \\
\text { ness training } \\
\text { mobile app } \\
(\text { Oiva })\end{array}$ & $\begin{array}{l}\text { Acceptance } \\
\text { and com- } \\
\text { mitment }\end{array}$ & $\begin{array}{l}\text { Improvement of } \\
\text { mental wellness and } \\
\text { reduction of stress } \\
\text { levels }\end{array}$ \\
\hline$\left|\begin{array}{l}\text { Anguera et al } \\
(2013)\end{array}\right|$ & $\begin{array}{l}\text { Effects of video } \\
\text { game training on } \\
\text { cognitive control of } \\
\text { older adults }\end{array}$ & $\begin{array}{l}46 \text { older } \\
\text { adults } \\
\mathrm{M}=67.5 \\
\text { years }\end{array}$ & $\begin{array}{l}\text { 3D Video } \\
\text { game }(\mathrm{Neu} \text { - } \\
\text { roRacer })\end{array}$ & $\begin{array}{l}\text { Multitasking } \\
\text { training }\end{array}$ & $\begin{array}{l}\text { Improvement of } \\
\text { sustained attention } \\
\text { and working } \\
\text { memory }\end{array}$ \\
\hline $\begin{array}{l}\text { Ball, Ed- } \\
\text { wards \& } \\
\text { Ross (2007) }\end{array}$ & $\begin{array}{l}\text { Effect of speed } \\
\text { processing training } \\
\text { on cognitive and } \\
\text { everyday functions }\end{array}$ & $\begin{array}{l}2,039 \text { older } \\
\text { adults } \\
\begin{array}{l}\mathrm{M}=73.94 \\
\text { years }\end{array} \\
\end{array}$ & $\begin{array}{l}\text { Computer- } \\
\text { based non- } \\
\text { verbal exer- } \\
\text { cises }\end{array}$ & $\begin{array}{l}\text { Speed of } \\
\text { processing } \\
\text { training }\end{array}$ & $\begin{array}{l}\text { Improvement on } \\
\text { executive function } \\
\text { and memory }\end{array}$ \\
\hline $\begin{array}{l}\text { Ballestı } \\
\text { al (201 }\end{array}$ & $\begin{array}{l}\text { Effect of training } \\
\text { older adults with } \\
\text { cognitive-based } \\
\text { video games }\end{array}$ & \begin{tabular}{|l|}
0 older \\
adults \\
$\mathrm{M}=28.8$ \\
years
\end{tabular} & $\begin{array}{l}\text { Web-based } \\
\text { tools }\end{array}$ & $\mid \begin{array}{l}\text { Cognitive- } \\
\text { based games }\end{array}$ & $\begin{array}{l}\text { Improvement in } \\
\text { processing speed, } \\
\text { attention and visual } \\
\text { memory }\end{array}$ \\
\hline $\begin{array}{l}\text { Chittaro \& } \\
\text { Sioni (2014) }\end{array}$ & $\begin{array}{l}\text { Evaluation of mobile } \\
\text { breathing training } \\
\text { apps }\end{array}$ & $\begin{array}{l}68 \text { adults } \\
M=24.73 \\
\text { years }\end{array}$ & $\begin{array}{l}\text { Adaptation } \\
\text { of breathing } \\
\text { frequency via } \\
\text { mobile app }\end{array}$ & $\begin{array}{l}\text { Breathing } \\
\text { training }\end{array}$ & $\begin{array}{l}\text { Reduction of stress } \\
\text { levels and possible } \\
\text { improvement of } \\
\text { well-being }\end{array}$ \\
\hline $\begin{array}{l}\text { Clemenson } \\
\text { and Stark } \\
(2015)\end{array}$ & $\begin{array}{l}\text { Effect of environ- } \\
\text { mental enrichment } \\
\text { via video games on } \\
\text { memory }\end{array}$ & $\begin{array}{l}68 \text { univer- } \\
\text { sity stu- } \\
\text { dents }\end{array}$ & Video games & $\begin{array}{l}\text { Environmen } \\
\text { tal enrich- } \\
\text { ment }\end{array}$ & $\begin{array}{l}\text { 3D video games } \\
\text { improved hippo- } \\
\text { campal-associated } \\
\text { memory }\end{array}$ \\
\hline $\begin{array}{l}\text { Craske et al, } \\
2009\end{array}$ & $\begin{array}{l}\text { Effect of web- } \\
\text { assisted cognitive } \\
\text { behavioral therapy } \\
\text { on anxiety disorders }\end{array}$ & \begin{tabular}{|l|}
13 clinics \\
with anxie- \\
ty disorders \\
$\begin{array}{l}25 \text { to } 59 \\
\text { years }\end{array}$ \\
\end{tabular} & $\begin{array}{l}\text { Web-based } \\
\text { tools }\end{array}$ & $\begin{array}{l}\text { Cognitive } \\
\text { behavioral } \\
\text { therapy }\end{array}$ & $\begin{array}{l}\text { Reduction of anxiety } \\
\text { symptoms }\end{array}$ \\
\hline \begin{tabular}{|l|} 
Fisher, Hol- \\
land, \\
Merzenich \& \\
Vinogradov \\
$(2009)$ \\
\end{tabular} & $\begin{array}{l}\text { Effect of computer- } \\
\text { ized cognitive train- } \\
\text { ing on patients with } \\
\text { schizophrenia }\end{array}$ & $\begin{array}{l}29 \text { adults } \\
\text { with schiz- } \\
\text { ophrenia } \\
\begin{array}{l}\mathrm{M}=42.86 \\
\text { years }\end{array} \\
\end{array}$ & $\begin{array}{l}\text { Computer- } \\
\text { based treat- } \\
\text { ment }\end{array}$ & $\begin{array}{l}\text { Auditory } \\
\text { training }\end{array}$ & $\begin{array}{l}\text { Improvement of } \\
\text { verbal working } \\
\text { memory and global } \\
\text { cognition }\end{array}$ \\
\hline \begin{tabular}{|l|} 
Kühn, \\
Gleich, \\
Lorenz, \\
Lindenberger \\
\& Gallinat \\
(2014) \\
\end{tabular} & $\begin{array}{l}\text { Effect of 3D video } \\
\text { game training on } \\
\text { structural brain } \\
\text { plasticity }\end{array}$ & $\begin{array}{l}\mathrm{M}=24.1 \\
\text { years }\end{array}$ & $\begin{array}{l}\text { Commercial } \\
\text { video game } \\
\text { (Super Mar- } \\
\text { io) }\end{array}$ & $\begin{array}{l}\text { 3D video } \\
\text { game with } \\
\text { orientation } \\
\text { and strategic } \\
\text { demands }\end{array}$ & $\begin{array}{l}\text { Increase of gray } \\
\text { matter on crucial } \\
\text { brain regions }\end{array}$ \\
\hline $\begin{array}{l}\text { Li, Ngo, } \\
\text { Nguyen \& } \\
\text { Levi (2011) }\end{array}$ & $\begin{array}{l}\text { Effect of playing } \\
\text { video-games on } \\
\text { spatial vision of } \\
\text { adults with amblyo- } \\
\text { pia }\end{array}$ & $\begin{array}{l}20 \text { adults } \\
\text { with am- } \\
\text { blyopia }\end{array}$ & $\begin{array}{l}\text { Playing } \\
\text { action video } \\
\text { games }\end{array}$ & $\begin{array}{l}\text { Tracking fi } \\
\text { moving } \\
\text { objects }\end{array}$ & $\begin{array}{l}\text { Plasticity in the } \\
\text { visual system }\end{array}$ \\
\hline Mishra, & Effect of online & 31 childre & Online cog- & Signal-to- & Improvement of \\
\hline
\end{tabular}




\begin{tabular}{|c|c|c|c|c|c|}
\hline \multirow{2}{*}{\begin{tabular}{|l} 
Sagar, Jo- \\
seph, Gaz- \\
zaley \& \\
Merzenich \\
$(2016)$ \\
\end{tabular}} & \multirow{2}{*}{$\begin{array}{l}\text { cognitive training on } \\
\text { cognitive measures } \\
\text { of children with } \\
\text { ADHD }\end{array}$} & $\begin{array}{l}\text { with } \\
\text { ADHD }\end{array}$ & \multirow[t]{2}{*}{\begin{tabular}{|l|} 
nitive exer- \\
cise modules
\end{tabular}} & \multirow{2}{*}{$\begin{array}{l}\text { noise resolu- } \\
\text { tion training }\end{array}$} & \multirow{2}{*}{$\begin{array}{l}\text { response inhibition } \\
\text { and executive func- } \\
\text { tion }\end{array}$} \\
\hline & & $\begin{array}{l}\mathrm{M}=12 \\
\text { years }\end{array}$ & & & \\
\hline \multirow{2}{*}{$\begin{array}{l}\text { Noorhida- } \\
\text { wati, } \\
\text { Ghalebandi } \\
\text { \& Hajar } \\
\text { (2015) }\end{array}$} & \multirow[b]{2}{*}{$\begin{array}{l}\text { Effect of children's } \\
\text { engagement with } \\
\text { mobile apps on the } \\
\text { learning process }\end{array}$} & Idren & \multirow[b]{2}{*}{$\begin{array}{l}\text { e-storybooks, } \\
\text { gaming and } \\
\text { creating apps }\end{array}$} & \multirow[b]{2}{*}{$\begin{array}{l}\text { Children's } \\
\text { engagement }\end{array}$} & \multirow[b]{2}{*}{$\begin{array}{l}\text { Enhancement of } \\
\text { awareness and goal } \\
\text { accomplishment }\end{array}$} \\
\hline & & 4-6 years & & & \\
\hline $\begin{array}{l}\text { Ordonez, } \\
\text { Yassuda \& }\end{array}$ & Digita & $\begin{array}{l}42 \text { older } \\
\text { adults }\end{array}$ & Computer & Digital & Improvement in \\
\hline $\begin{array}{l}\text { Cachioni } \\
\text { (2011) }\end{array}$ & older adults & $\begin{array}{l}\mathrm{M}=67.38 \\
\text { years }\end{array}$ & $\begin{array}{l}\text { training } \\
\text { workshops }\end{array}$ & $\begin{array}{l}\text { inclusion } \\
\text { classes }\end{array}$ & $\begin{array}{l}\text { language and } \\
\text { memory domain }\end{array}$ \\
\hline $\begin{array}{l}\text { Sánchez \& } \\
\text { Olivares } \\
(2011)\end{array}$ & $\begin{array}{l}\text { Impact of mobile } \\
\text { serious games on } \\
\text { problem solving } \\
\text { skills }\end{array}$ & $\begin{array}{l}2278^{\text {th }} \\
\text { grade } \\
\text { students }\end{array}$ & $\begin{array}{l}\text { Educational } \\
\text { video games }\end{array}$ & $\begin{array}{l}\text { Mobile } \\
\text { serious } \\
\text { games }\end{array}$ & $\begin{array}{l}\text { Improvement of } \\
\text { problem solving and } \\
\text { plan execution skills }\end{array}$ \\
\hline \multirow{2}{*}{$\begin{array}{l}\text { Smith et al } \\
(2009)\end{array}$} & \multirow{2}{*}{$\begin{array}{l}\text { Efficacy of a brain } \\
\text { plasticity-based } \\
\text { computerized train- } \\
\text { ing program in older } \\
\text { adults }\end{array}$} & $\begin{array}{l}242 \text { older } \\
\text { adults }\end{array}$ & \multirow{2}{*}{\begin{tabular}{|l|} 
Computer- \\
ized training \\
with auditory \\
information \\
processing \\
\end{tabular}} & \multirow{2}{*}{$\begin{array}{l}\text { Brain plas- } \\
\text { ticity }\end{array}$} & \multirow{2}{*}{$\begin{array}{l}\text { Improvement of } \\
\text { memory and atten- } \\
\text { tion }\end{array}$} \\
\hline & & $\begin{array}{l}\mathrm{M}=75.6 \\
\text { years }\end{array}$ & & & \\
\hline \multirow{2}{*}{\begin{tabular}{|l} 
Van \\
Muijden, \\
Band \& \\
Hommel \\
$(2012)$ \\
\end{tabular}} & \multirow{2}{*}{$\begin{array}{l}\text { Effect of online } \\
\text { cognitive training } \\
\text { games on healthy } \\
\text { older adults }\end{array}$} & $\begin{array}{l}54 \text { older } \\
\text { adults }\end{array}$ & \multirow{2}{*}{$\begin{array}{l}\text { Online cog- } \\
\text { nitive train- } \\
\text { ing games }\end{array}$} & \multirow{2}{*}{$\begin{array}{l}\text { Brain train- } \\
\text { ing game } \\
\text { intervention }\end{array}$} & \multirow{2}{*}{$\begin{array}{l}\text { Improvement of } \\
\text { inhibition and induc- } \\
\text { tive reasoning }\end{array}$} \\
\hline & & $\begin{array}{l}\mathrm{M}=67.8 \\
\text { years }\end{array}$ & & & \\
\hline $\begin{array}{l}\text { Zimmerman } \\
\text { \& Christakis } \\
(2005)\end{array}$ & $\begin{array}{l}\text { Evaluation of the } \\
\text { cognitive outcomes } \\
\text { from television } \\
\text { viewing }\end{array}$ & $\begin{array}{l}1,979 \\
\text { children \& } \\
\text { young } \\
\text { adults }\end{array}$ & $\begin{array}{l}\text { Television } \\
\text { viewing }\end{array}$ & & $\begin{array}{l}\text { Television viewing } \\
\text { had positive effect } \\
\text { on } 3 \text { to } 5 \text { year-old } \\
\text { children }\end{array}$ \\
\hline \multirow{2}{*}{$\begin{array}{l}\text { Shellington, } \\
\text { Felfeli, Shi- } \\
\text { gematsu, Gill } \\
\& \text { Petrella } \\
(2017) \\
\end{array}$} & \multirow{2}{*}{$\begin{array}{l}\text { Effect of square- } \\
\text { stepping exercise via } \\
\text { mobile app on older } \\
\text { adults cognitive } \\
\text { function }\end{array}$} & $\begin{array}{l}19 \text { older } \\
\text { adults }\end{array}$ & \multirow{2}{*}{$\begin{array}{l}\text { Smartphone } \\
\text { app } \\
\text { (Healthe- } \\
\text { Brain) }\end{array}$} & \multirow{2}{*}{$\begin{array}{l}\text { Physical } \\
\text { exercise }\end{array}$} & \multirow{2}{*}{$\begin{array}{l}\text { Improvement of } \\
\text { executive function- } \\
\text { ing }\end{array}$} \\
\hline & & $\begin{array}{l}\mathrm{M}=68.3 \\
\text { years }\end{array}$ & & & \\
\hline $\begin{array}{l}\text { Nan et al } \\
(2012)\end{array}$ & $\begin{array}{l}\text { Effect of neurofeed- } \\
\text { back training on } \\
\text { short-term memory }\end{array}$ & $\begin{array}{l}32 \text { students } \\
M=23.28 \\
\text { years }\end{array}$ & $\begin{array}{l}\text { Electroen- } \\
\text { cephalogram } \\
(\text { EEG) }\end{array}$ & $\begin{array}{l}\text { Alpha neu- } \\
\text { rofeedback } \\
\text { training }\end{array}$ & $\begin{array}{l}\text { Enhancement of } \\
\text { self-regulation and } \\
\text { short-term memory } \\
\text { performance }\end{array}$ \\
\hline $\begin{array}{l}\text { Wang and } \\
\text { Hsieh (2013) }\end{array}$ & $\begin{array}{l}\text { Effect of neurofeed- } \\
\text { back training on } \\
\text { older adults cogni- } \\
\text { tive performance }\end{array}$ & \begin{tabular}{|l|}
32 older \\
adults \\
$\begin{array}{l}\mathrm{M}=65 \\
\text { years }\end{array}$ \\
\end{tabular} & \begin{tabular}{|l|} 
Electroen- \\
cephalogram \\
(EEG)
\end{tabular} & $\begin{array}{l}\text { fm } \theta \text { uptrain- } \\
\text { ing }\end{array}$ & $\begin{array}{l}\text { Improvement of } \\
\text { attention and work- } \\
\text { ing memory }\end{array}$ \\
\hline \multirow{2}{*}{\begin{tabular}{|l|} 
Voelbel, \\
Ceceli, \\
Georgieva, \\
Tortarolo \& \\
Lindsey \\
(2014) \\
\end{tabular}} & \multirow{2}{*}{$\begin{array}{l}\text { Effect of computer- } \\
\text { ized cognitive train- } \\
\text { ing on adults with } \\
\text { traumatic brain } \\
\text { injury (TBI) }\end{array}$} & $\begin{array}{l}20 \text { adults } \\
\text { with TBI } \\
\end{array}$ & \multirow{2}{*}{$\begin{array}{l}\text { Structured } \\
\text { computerized } \\
\text { modules }\end{array}$} & \multirow[b]{2}{*}{$\begin{array}{l}\text { Cognitive } \\
\text { training }\end{array}$} & \multirow{2}{*}{$\begin{array}{l}\text { Improvement of } \\
\text { processing speed } \\
\text { and task switching }\end{array}$} \\
\hline & & $\begin{array}{l}\mathrm{M}=43.33 \\
\text { years }\end{array}$ & & & \\
\hline
\end{tabular}


Paper-Computerized Training for Neuroplasticity and Cognitive Improvement

\begin{tabular}{|c|c|c|c|c|c|}
\hline \multirow{2}{*}{$\begin{array}{l}\text { Subramania } \\
\text { m et al } \\
(2012)\end{array}$} & \multirow{2}{*}{$\begin{array}{l}\text { Effect of computer- } \\
\text { ized training on } \\
\text { schizophrenia's } \\
\text { neural activity }\end{array}$} & $\begin{array}{l}31 \text { schizo- } \\
\text { phrenia } \\
\text { patients } \\
\end{array}$ & \multirow{2}{*}{$\begin{array}{l}\text { Computer- } \\
\text { ized training }\end{array}$} & \multirow{2}{*}{$\begin{array}{l}\text { Audito- } \\
\text { ry/verbal, } \\
\text { visual and } \\
\text { social pro- } \\
\text { cessing } \\
\text { exercises }\end{array}$} & \multirow{2}{*}{$\begin{array}{l}\text { Improvement of } \\
\text { reality monitoring } \\
\text { and increase in } \\
\text { mPFC activation }\end{array}$} \\
\hline & & $\begin{array}{l}\mathrm{M}=40 \\
\text { years }\end{array}$ & & & \\
\hline
\end{tabular}

\section{Discussion}

This review aimed to evaluate the impact of computerized training on cognitive improvement of healthy or cognitive impaired individuals. Literature review of the decade indicated that Information and Communication Technologies, such as computer-based or web-based training tools, commercial or serious games, training mobile apps, as well as neurofeedback devices could be used for intervention programs including cognitive training [50]. In addition, there is evidence that cognitive training techniques such as cognitive behavioural therapy, speed of processing training and neurofeedback training, as well as traditional techniques as physical activity, breathing training and eye tracking could significantly enhance cognitive improvement both for healthy as for cognitive impaired individuals. Furthermore, neuroimaging studies provide evidence of brain plasticity from birth to late adulthood, indicating that cognitive skills are trainable throughout life [51]. However, most Information and Communication training tools are still at an experimental level, indicating that further research is required before the universal application of these tools for reliable cognitive improvement.

\section{References}

[1] Bryck, R. L., \& Fisher, P. A. (2012). Training the brain: practical applications of neural plasticity from the intersection of cognitive neuroscience, developmental psychology, and prevention science. American Psychologist, 67(2), 87. https://doi.org/10.1037/a0024657

[2] Faubert, J., \& Sidebottom, L. (2012). Perceptual-cognitive training of athletes. Journal of Clinical Sport Psychology, 6(1), 85-102. https://doi.org/10.1123/jcsp.6.1.85

[3] Green, C. S., \& Bavelier, D. (2008). Exercising your brain: a review of human brain plasticity and training-induced learning. Psychology and aging, 23(4), 692. https://doi.org/ $10.1037 / \mathrm{a} 0014345$

[4] Zelinski, E. M., \& Reyes, R. (2009). Cognitive benefits of computer games for older adults. Gerontechnology: international journal on the fundamental aspects of technology to serve the ageing society, 8(4), 220. https://doi.org/10.4017/gt.2009.08.04.004.00

[5] Belleville, S., Clement, F., Mellah, S., Gilbert, B., Fontaine, F., \& Gauthier, S. (2011). Training-related brain plasticity in subjects at risk of developing Alzheimer's disease. Brain, 134(6), 1623-1634. https://doi.org/10.1093/brain/awr037

[6] Kleim, J. A., \& Jones, T. A. (2008). Principles of experience-dependent neural plasticity: implications for rehabilitation after brain damage. Journal of speech, language, and hearing research, 51(1), S225-S239. https://doi.org/10.1044/1092-4388(2008/018) 
[7] Sehm, B., Taubert, M., Conde, V., Weise, D., Classen, J., Dukart, J., . \& Ragert, P. (2014). Structural brain plasticity in Parkinson's disease induced by balance training. Neurobiology of aging, 35(1), 232-239. https://doi.org/10.1016/j.neurobiolaging.2013.06.021

[8] Spironelli, C., \& Angrilli, A. (2015). Brain plasticity in aphasic patients: intra-and interhemispheric reorganisation of the whole linguistic network probed by N150 and N350 components. Scientific Reports, 5, 12541. https://doi.org/10.1038/srep12541

[9] Herholz, S. C., \& Zatorre, R. J. (2012). Musical training as a framework for brain plasticity: behavior, function, and structure. Neuron, 76(3), 486-502. https://doi.org/10.10 16/j.neuron.2012.10.011

[10] Wan, C. Y., \& Schlaug, G. (2010). Music making as a tool for promoting brain plasticity across the life span. The Neuroscientist, 16(5), 566-577. https://doi.org/10.1177/10738584 10377805

[11] Erickson, K. I., Gildengers, A. G., \& Butters, M. A. (2013). Physical activity and brain plasticity in late adulthood. Dialogues in clinical neuroscience, 15(1), 99.

[12] Nagamatsu, L. S., Handy, T. C., Hsu, C. L., Voss, M., \& Liu-Ambrose, T. (2012). Resistance training promotes cognitive and functional brain plasticity in seniors with probable mild cognitive impairment. Archives of internal medicine, 172(8), 666-668. https://doi.org/10.1001/archinternmed.2012.379

[13] Pareja-Galeano, H., Brioche, T., Sanchis-Gomar, F., Montal, A., Jovaní, C., MartínezCosta, C. \& Vina, J. (2013). Impact of exercise training on neuroplasticity-related growth factors in adolescents. J Musculoskelet Neuronal Interact, 13(3), 368-71.

[14] Baijal, S., \& Gupta, R. (2008). Meditation-based training: a possible intervention for attention deficit hyperactivity disorder. Psychiatry (Edgmont), 5(4), 48.

[15] Zimmerman, F. J., \& Christakis, D. A. (2005). Children's television viewing and cognitive outcomes: a longitudinal analysis of national data. Archives of Pediatrics \& Adolescent Medicine, 159(7), 619-625. https://doi.org/10.1001/archpedi.159.7.619

[16] Zimmerman, F. J., Christakis, D. A., \& Meltzoff, A. N. (2007). Associations between media viewing and language development in children under age 2 years. The Journal of pediatrics, 151(4), 364-368. https://doi.org/10.1016/j.jpeds.2007.04.071

[17] Mar, R. A., Tackett, J. L., \& Moore, C. (2010). Exposure to media and theory-of-mind development in preschoolers. Cognitive Development, 25(1), 69-78. https://doi.org/10.10 16/j.cogdev.2009.11.002

[18] Johnson, J. G., Cohen, P., Kasen, S., \& Brook, J. S. (2007). Extensive television viewing and the development of attention and learning difficulties during adolescence. Archives of pediatrics \& adolescent medicine, 161(5), 480-486. https://doi.org/10.1001/archpedi.161. $\underline{5.480}$

[19] Craske, M. G., Rose, R. D., Lang, A., Welch, S. S., Campbell-Sills, L., Sullivan, G., . \& Roy-Byrne, P. P. (2009). Computer-assisted delivery of cognitive behavioral therapy for anxiety disorders in primary-care settings. Depression and anxiety, 26(3), 235-242. https://doi.org/10.1002/da.20542

[20] Mishra, J., Sagar, R., Joseph, A. A., Gazzaley, A., \& Merzenich, M. M. (2016). Training sensory signal-to-noise resolution in children with ADHD in a global mental health setting. Translational psychiatry, 6(4), e781. https://doi.org/10.1038/tp.2016.45

[21] Ballesteros, S., Prieto, A., Mayas, J., Toril, P., Pita, C., Ponce de León, L.,. \& Waterworth, J. (2014). Brain training with non-action video games enhances aspects of cognition in older adults: a randomized controlled trial. Frontiers in aging neuroscience, 6, 277. https://doi.org/10.3389/fnagi.2015.00082 
[22] Ordonez, T. N., Yassuda, M. S., \& Cachioni, M. (2011). Elderly online: effects of a digital inclusion program in cognitive performance. Archives of Gerontology and Geriatrics, 53(2), 216-219. https://doi.org/10.1016/j.archger.2010.11.007

[23] Ball, K., Edwards, J. D., \& Ross, L. A. (2007). The impact of speed of processing training on cognitive and everyday functions. The Journals of Gerontology Series B: Psychological Sciences and Social Sciences, 62(Special_Issue_1), 19-31. https://doi.org/10.1093/geronb/ 62.special issue 1.19

[24] Smith, G. E., Housen, P., Yaffe, K., Ruff, R., Kennison, R. F., Mahncke, H. W., \& Zelinski, E. M. (2009). A cognitive training program based on principles of brain plasticity: results from the Improvement in Memory with Plasticity-based Adaptive Cognitive Training (IMPACT) Study. Journal of the American Geriatrics Society, 57(4), 594-603. https://doi.org/10.1111/j.1532-5415.2008.02167.x

[25] Fisher, M., Holland, C., Merzenich, M. M., \& Vinogradov, S. (2009). Using neuroplasticity-based auditory training to improve verbal memory in schizophrenia. American Journal of Psychiatry, 166(7), 805-811. https://doi.org/10.1176/appi.ajp.2009.08050757

[26] Voelbel, G., Ceceli, A., Georgieva, S., Tortarolo, C., \& Lindsey, H. (2014). C-40 Computerized Neuroplasticity Training Increases Processing Speed of Verbal Information: A Pilot Study of Adults with Traumatic Brain Injury. Archives of Clinical Neuropsychology, 29(6), 589-589. https://doi.org/10.1093/arclin/acu038.221

[27] Subramaniam, K., Luks, T. L., Fisher, M., Simpson, G. V., Nagarajan, S., \& Vinogradov, S. (2012). Computerized cognitive training restores neural activity within the reality monitoring network in schizophrenia. Neuron, 73(4), 842-853. https://doi.org/10.1016/j. neuron.2011.12.024

[28] Clemenson, G. D., \& Stark, C. E. (2015). Virtual environmental enrichment through video games improves hippocampal-associated memory. Journal of Neuroscience, 35(49), 16116-16125. https://doi.org/10.1523/jneurosci.2580-15.2015

[29] Blumberg, F. C., \& Fisch, S. M. (2013). Introduction: Digital games as a context for cognitive development, learning, and developmental research. New directions for child and adolescent development, 2013(139), 1-9. https://doi.org/10.1002/cad.20026

[30] Kühn, S., Gleich, T., Lorenz, R. C., Lindenberger, U., \& Gallinat, J. (2014). Playing Super Mario induces structural brain plasticity: gray matter changes resulting from training with a commercial video game. Molecular psychiatry, 19(2), 265. https://doi.org/10.1038/mp .2013 .120

[31] Anguera, J. A., Boccanfuso, J., Rintoul, J. L., Al-Hashimi, O., Faraji, F., Janowich, J., . \& Gazzaley, A. (2013). Video game training enhances cognitive control in older adults. Nature, 501(7465), 97. https://doi.org/10.1038/nature12486

[32] Van Muijden, J., Band, G. P., \& Hommel, B. (2012). Online games training aging brains: limited transfer to cognitive control functions. Frontiers in human neuroscience, 6, 221. https://doi.org/10.3389/fnhum.2012.00221

[33] Li, R. W., Ngo, C., Nguyen, J., \& Levi, D. M. (2011). Video-game play induces plasticity in the visual system of adults with amblyopia. PLoS biology, 9(8), e1001135. https://doi. org/10.1371/journal.pbio.1001135

[34] Zhao, J., Freeman, B., \& Li, M. (2016). Can mobile phone apps influence people's health behavior change? An evidence review. Journal of medical Internet research, 18(11). https://doi.org/10.2196/jmir.5692

[35] Noorhidawati, A., Ghalebandi, S. G., \& Hajar, R. S. (2015). How do young children engage with mobile apps? Cognitive, psychomotor, and affective perspective. Computers \& Education, 87, 385-395. https://doi.org/10.1016/j.compedu.2015.07.005 
[36] Torous, J., Staples, P., Fenstermacher, E., Dean, J., \& Keshavan, M. (2016). Barriers, benefits, and beliefs of brain training smartphone apps: an internet survey of younger US consumers. Frontiers in human neuroscience, 10, 180. https://doi.org/10.3389/fnhum.20 $\underline{16.00253}$

[37] Ahtinen, A., Mattila, E., Välkkynen, P., Kaipainen, K., Vanhala, T., Ermes, M., . \& Lappalainen, R. (2013). Mobile mental wellness training for stress management: feasibility and design implications based on a one-month field study. JMIR mHealth and uHealth, 1(2). https://doi.org/10.2196/mhealth.2596

[38] Sánchez, J., \& Olivares, R. (2011). Problem solving and collaboration using mobile serious games. Computers \& Education, 57(3), 1943-1952. https://doi.org/10.1016/j.comp edu.2011.04.012

[39] Shellington, E. M., Felfeli, T., Shigematsu, R., Gill, D. P., \& Petrella, R. J. (2017). HealtheBrain: an innovative smartphone application to improve cognitive function in older adults. Mhealth, 3. https://doi.org/10.21037/mhealth.2017.04.05

[40] Chittaro, L., \& Sioni, R. (2014). Evaluating mobile apps for breathing training: The effectiveness of visualization. Computers in Human Behavior, 40, 56-63. https://doi.org/ 10.1016/j.chb.2014.07.049

[41] Escolano, C., Aguilar, M., \& Minguez, J. (2011, August). EEG-based upper alpha neurofeedback training improves working memory performance. In Engineering in medicine and biology society, EMBC, 2011 Annual International Conference of the IEEE (pp. 23272330). IEEE. https://doi.org/10.1109/iembs.2011.6090651

[42] Perreau-Linck, E., Lessard, N., Lévesque, J., \& Beauregard, M. (2010). Effects of neurofeedback training on inhibitory capacities in ADHD children: a single-blind, randomized, placebo-controlled study. Journal of Neurotherapy, 14(3), 229-242. https://doi.org/10.1080/10874208.2010.501514

[43] Pineda, J. A., Brang, D., Hecht, E., Edwards, L., Carey, S., Bacon, M., . \& Rork, A. (2008). Positive behavioral and electrophysiological changes following neurofeedback training in children with autism. Research in Autism Spectrum Disorders, 2(3), 557-581. https://doi.org/10.1016/j.rasd.2007.12.003

[44] Rossi-Izquierdo, M., Ernst, A., Soto-Varela, A., Santos-Pérez, S., Faraldo-García, A., Sesar-Ignacio, Á., \& Basta, D. (2013). Vibrotactile neurofeedback balance training in patients with Parkinson's disease: reducing the number of falls. Gait \& Posture, 37(2), 195 200. https://doi.org/10.1016/j.gaitpost.2012.07.002

[45] Nan, W., Rodrigues, J. P., Ma, J., Qu, X., Wan, F., Mak, P. I., . \& Rosa, A. (2012). Individual alpha neurofeedback training effect on short term memory. International journal of psychophysiology, 86(1), 83-87.https://doi.org/10.1016/j.ijpsycho.2012.07.182

[46] Wang, J. R., \& Hsieh, S. (2013). Neurofeedback training improves attention and working memory performance. Clinical Neurophysiology, 124(12), 2406-2420. https://doi.org/10. 1016/j.clinph.2013.05.020

[47] Ghaziri, J., Tucholka, A., Larue, V., Blanchette-Sylvestre, M., Reyburn, G., Gilbert, G., . $\&$ Beauregard, M. (2013). Neurofeedback training induces changes in white and gray matter. Clinical EEG and Neuroscience, 44(4), 265-272. https://doi.org/10.1177/15500594 $\underline{13476031}$

[48] Sitaram, R., Ros, T., Stoeckel, L., Haller, S., Scharnowski, F., Lewis-Peacock, J., \& Birbaumer, N. (2017). Closed-loop brain training: the science of neurofeedback. Nature Reviews Neuroscience, 18(2), 86. https://doi.org/10.1038/nrn.2016.164

[49] Zoefel, B., Huster, R. J., \& Herrmann, C. S. (2011). Neurofeedback training of the upper alpha frequency band in EEG improves cognitive performance. Neuroimage, 54(2), 14271431. https://doi.org/10.1016/j.neuroimage.2010.08.078 
[50] Drigas, A., \& Karyotaki, M. (2014). Learning Tools and Applications for Cognitive Improvement. International Journal of Engineering Pedagogy (iJEP), 4(3), 71-77. https://doi.org/10.3991/ijep.v4i3.3665

[51] Pappas, M., Drigas, A., Malli, E., \& Kalpidi, V. (2018). Enhanced Assessment Technology and Neurocognitive Aspects of Specific Learning Disorder with Impairment in Mathematics. International Journal of Engineering Pedagogy, 8(1), 4-15. https://doi.org/10.3991/ ijep.v8i1.7370

\section{$7 \quad$ Authors}

Marios A. Pappas is a Research Associate at National Center for Scientific Research "Demokritos", Institute of Informatics and Telecommunications - Net Media Lab \& Mind-Brain R\&D, Agia Paraskevi 153 10, Athens, Greece

Athanasios S. Drigas is a Director of Research at National Center for Scientific Research 'Demokritos', Institute of Informatics and Telecommunications, and a Coordinator of Net Media Lab \& Brain-Mind R\&D, Agia Paraskevi, 153 10, Athens, Greece E-mail: dr@iit.demokritos.gr

Article submitted 2019-02-08. Resubmitted 2019-05-21. Final acceptance 2019-05-27. Final version published as submitted by the authors. 